Draft VERSion, OCtOBER 25, 2018

Preprint typeset using $\mathrm{L}^{\mathrm{A}} \mathrm{T}_{\mathrm{E}} \mathrm{X}$ style emulateapj v. 11/10/09

\title{
VELOCITY ANISOTROPY AS A DIAGNOSTIC OF THE MAGNETIZATION OF THE INTERSTELLAR MEDIUM AND MOLECULAR CLOUDS
}

\author{
A. Esquivel ${ }^{1} \&$ A. LAzARian ${ }^{2}$ \\ ${ }^{1}$ Instituto de Ciencias Nucleares, Universidad Nacional Autónoma de México, \\ Apartado Postal 70-543, 04510 México D.F., México \\ ${ }^{2}$ Astronomy Department, University of Wisconsin-Madison, 475 N. Charter Street, Madison, WI 53706-1582, USA \\ Draft version, October 25, 2018
}

\begin{abstract}
We use a set of magnetohydrodynamics (MHD) simulations of fully-developed (driven) turbulence to study the anisotropy in the velocity field that is induced by the presence of the magnetic field. In our models we study turbulence characterized by sonic Mach numbers $M_{s}$ from 0.7 to 7.5 , and Alfvén Mach numbers from 0.4 to 7.7. These are used to produce synthetic observations (centroid maps) that are analyzed. To study the effect of large scale density fluctuations and of white noise we have modified the density fields and obtained new centroid maps, which are analyzed. We show that restricting the range of scales at which the anisotropy is measured makes the method robust against such fluctuations. We show that the anisotropy in the structure function of the maps reveals the direction of the magnetic field for $M_{A} \lesssim 1.5$, regardless of the sonic Mach number. We found that the degree of anisotropy can be used to determine the degree of magnetization (i.e. $M_{A}$ ) for $M_{A} \lesssim 1.5$. To do this, one needs an additional measure of the sonic Mach number and an estimate of the LOS magnetic field, both feasible by other techniques, offering a new opportunity to study the magnetization state of the interstellar medium.
\end{abstract}

Subject headings: ISM: general — ISM: structure — magnetohydrodynamics (MHD) — radio lines: ISM - turbulence

\section{INTRODUCTION}

It is well accepted that turbulence plays a central role in the dynamics and transport phenomena in the interstellar medium (ISM). For an overview we refer the reader to the recent reviews by Elmegreen \& Scalo (2004); Mac Low \& Klessen (2004); Ballesteros-Paredes et al. (2007); McKee \& Ostriker (2007), and references therein. Moreover, turbulence in the ISM is magnetized, so in order to understand its properties one has to address those of the magnetic field as well.

The presence of a magnetic field introduces a preferential direction of motion for the charged particles and in consequence makes the turbulent cascade anisotropic, which has been known for some time now (Montgomery 1982 Higdon 1984). In a turbulent magnetized medium the kinetic energy of large-scale motions is larger than those at small-scales, but the local magnetic field (thus the magnetic energy) is comparable at all scales. Therefore at large-scales the magnetic field does not dominate dynamically, but it becomes more important as we go into smaller scales. The result are elongated eddies, which become more elongated as the energy cascade goes down to smaller scales (Cho \& Vishniac 2000: Maron \& Goldreich 2001; Cho, Lazarian, \& Vishniac 2002, henceforth CLV02). Recent decades have seen important progress in the understanding of the magnetic turbulence. A pioneering study is the self-consistent model of magnetohydrodynamic (MHD) turbulence of Goldreich \& Sridhar (1995, hereafter GS95) where the concept of a scale dependent anisotropy is built in ${ }^{1}$, The

\footnotetext{
esquivel@nucleares.unam.mx, lazarian@astro.wisc.edu

1 The initial publication did not have the concept of the local magnetic field built in. In fact, the closure relations in the paper
}

GS95 model was later supported by the numerical simulations of Cho \& Vishniac (2000); Maron \& Goldreich (2001); CLV02 . The ideas on compressible turbulence sketched in GS95 have proved profound, and have been supported and developed in further studies (Lithwick \& Goldreich 2001; Cho \& Lazarian 2003; Kowal \& Lazarian 2010). While some aspects of GS95 scaling were claimed to be controversial and not consistent with numerical simulations, e.g. the predicted Kolmogorov-type index of the spectrum, later studies (e.g. Beresnyak \& Lazarian 2010) have revealed the limitations of the numerical simulations which induced these controversies. All in all, we believe that the GS95 model (with the adjustments and improvements introduced in subsequent publications) provides the best representation of the MHD turbulence statistics.

The GS95 model is well described in many reviews (see Lazarian \& Cho 2005) and we do not dwell upon its details. We just mention that the qualitative understanding of the model can be obtained if one assumes that mixing motions perpendicular to the local magnetic field create a turbulent Kolmogorov-like cascade. The eddies are elongated along the local direction of magnetic field and the relation between the parallel and perpendicular scales of the eddies is given by the so-called "critically balance," which is reflected in the equality of the eddy turnover time and the timescale of propagation of Alfvén waves along the magnetic field of the eddy. The tensor describing the turbulent magnetic field is presented in

were written in the mean field frame of reference. The correct understanding that the eddies can be described only in the system of reference related to the local magnetic field of the eddies in question was obtained later (Lazarian \& Vishniac 1999 Cho \& Vishniac 2000 Maron \& Goldreich 2001 (LVO2) 


\section{CLV02}

The underlying anisotropies of turbulence result in the anisotropies of the observed statistics of turbulence. However, the anisotropy of interstellar turbulence which is accessible to the observer averaging emission along the line of sight crossing a turbulent volume is different from the GS95 predictions. In the "global" system of reference, related to the mean magnetic field, the anisotropy is no longer-scale dependent but is determined by the anisotropy of the largest eddies. A suggestion of using such anisotropy to study turbulence and the direction of the mean magnetic field was made Lazarian, Pogosyan, \& Esquivel (2002, henceforth LPE02) , where the feasibility of such studies was illustrated with synthetic spectral line emission maps obtained via MHD turbulence simulations. The anisotropy is readily evident from twopoint statistics (e.g. correlation/structure functions and power-spectra), where more power (larger dispersion) is concentrated in the direction perpendicular to the magnetic field (see for instance Esquivel et al. 2003; Vestuto et al. 2003). The aforementioned studies provided the framework for the observational studies in Heyer et al. (2008).

In this paper we revisit the velocity anisotropy from numerical simulations and synthetic observations. In particular, we study how such anisotropy depends on the global Alfvénic and sonic Mach numbers of the turbulence.

If one knows the Alfvén Mach number, then by knowing the turbulent velocity dispersion, an estimation of the media magnetization (i.e. its Alfvén speed) is possible. The determination of the Alfén speed, which is a crucial parameter for theoretical and numerical models, has been a great challenge for current techniques that probe the ISM.

We describe our MHD simulations in $\S 2$, and the way of measuring velocity anisotropy in observations in $\S 3$. Results of our study, namely the degree of anisotropy obtained as a function of Alfvénic and sonic Mach numbers are presented in $\S 4$. We finish with a discussion and provide a summary in $\S 5$.

\section{MHD MODELS}

We use a set of three-dimensional MHD simulations of fully-developed (driven) turbulence to produce maps of velocity centroids and of the average LOS velocity ${ }^{2}$

The simulations presented in this work were obtained solving the ideal MHD equations in a periodic box,

$$
\begin{aligned}
\frac{\partial \rho}{\partial t}+\nabla \cdot(\rho \mathbf{v}) & =0 \\
\frac{\partial \rho \mathbf{v}}{\partial t}+\nabla \cdot\left[\rho \mathbf{v} \mathbf{v}+\left(p+\frac{B^{2}}{8 \pi}\right) \mathbf{I}-\frac{1}{4 \pi} \mathbf{B B}\right] & =\mathbf{f}, \\
\frac{\partial \mathbf{B}}{\partial t}-\nabla \times(\mathbf{v} \times \mathbf{B}) & =\mathbf{0},
\end{aligned}
$$

with an isothermal equation of state $\left(p=c_{s}^{2} \rho\right.$, where $p$ is the gas pressure, $c_{s}$ the sound speed, and $\rho$ the mass den-

\footnotetext{
2 The average LOS velocity traces the statistics of velocity in the same manner that column density traces density. However, it can not be obtained directly from observations, while column density can. In previous works we have termed it "integrated velocity" (e.g. Lazarian \& Esquivel 2003 Esquivel \& Lazarian 2005).
}

TABLE 1

Parameters of the MHD simulations.

\begin{tabular}{lccccc}
\hline \hline Model & $v_{A, 0}$ & $\left\langle P_{\text {gas }, 0}\right\rangle$ & $M_{s}$ & $M_{A}$ & Resolution \\
\hline M1 & 0.1 & 0.01 & $\sim 7.6$ & $\sim 7.6$ & $512^{3}$ \\
M2 & 0.1 & 0.1 & $\sim 2.4$ & $\sim 7.7$ & $512^{3}$ \\
M3 & 0.1 & 1.0 & $\sim 0.8$ & $\sim 7.7$ & $512^{3}$ \\
M4 & 0.5 & 0.01 & $\sim 7.5$ & $\sim 1.5$ & $256^{3}$ \\
M5 & 0.5 & 0.1 & $\sim 2.3$ & $\sim 1.5$ & $256^{3}$ \\
M6 & 0.5 & 1.0 & $\sim 0.7$ & $\sim 1.4$ & $256^{3}$ \\
M7 & 1.0 & 0.01 & $\sim 7.4$ & $\sim 0.7$ & $512^{3}$ \\
M8 & 1.0 & 0.1 & $\sim 2.3$ & $\sim 0.7$ & $512^{3}$ \\
M9 & 1.0 & 1.0 & $\sim 0.7$ & $\sim 0.7$ & $512^{3}$ \\
M10 & 2.5 & 0.01 & $\sim 9.8$ & $\sim 0.4$ & $256^{3}$ \\
M11 & 2.5 & 0.1 & $\sim 3.3$ & $\sim 0.4$ & $256^{3}$ \\
M12 & 2.5 & 1.0 & $\sim 1.1$ & $\sim 0.4$ & $256^{3}$ \\
\hline
\end{tabular}

sity) and the additional constraint of $\nabla \cdot \mathbf{B}=0$, achieved with a constrained transport (CT) algorithm (see e.g. Tóth 2000). The integration method is a second-orderaccurate hybrid essentially nonoscillatory (ENO) scheme (see Cho \& Lazarian 2002). In order to avoid spurious osscilations, the code switches from a ENO weighted scheme (Jiang \& Wu 1999, where variables are smooth) to a convex ENO scheme (Shu \& Osher 1989, where strong gradients are found). 'The time is marched with a two stage Runge-Kutta method. The source term at the right hand side of equation (2) is a large-scale pseudo-random driving. Such driving is purely solenoidal and is performed in Fourier space at a fixed wave number $k=2.5$ (a scale of $1 / 2.5$ of the computational domain). For details about the driving see Cho \& Lazarian (2003) and Kowal et al. (2007). Initially $\rho=1$ and the Alfvén speed $v_{A}=|\mathbf{B}| / \sqrt{4 \pi \rho}$ depends on the model. In stationary state the rms velocity is also close to unity $\left(v_{\text {rms }} \sim 0.7\right)$. The parameters that define the experiments used are the sonic and Alfvénic Mach numbers at the injection scale $M_{s} \equiv\left\langle V_{L} / c_{s}\right\rangle$ and $M_{A} \equiv\left\langle V_{L} / v_{A}\right\rangle$, respectively, where $V_{L}=v_{\text {rms }}$ is the turbulent velocity at the injection scale, and $\langle\ldots\rangle$ stands for average over the entire computational domain. These parameters can be controlled by the values of the gas pressure and the Alvfén speed at the beginning of the simulation, $\left\langle P_{\text {gas }, 0}\right\rangle$ and $v_{A, 0}$, respectively. In Table 1 we list the numerical experiments that cover sub-sonic and super-sonic regimes, combined with different intensities of magnetic field (yielding sub-Alfvénic and super-Alfvénic regimes as well). We have to mention that the value of $v_{r m s}$ in stationary state varies from model to model, the Mach numbers presented in the table are measured from the output of the simulations.

The initial magnetic field is of the form $\mathbf{B}=\mathbf{B}_{\text {ext }}+\mathbf{b}$, a uniform field $\mathbf{B}_{\text {ext }}$ plus a fluctuating part $\mathbf{b}$. Initially, $\mathbf{b}=0$ and $\mathbf{B}_{\text {ext }}$ is aligned in the $x$-direction. When the simulations reach a stationary state the magnitude of the mean and the fluctuating parts are of the same order, while the mean magnetic field remains aligned with the $x$-axis.

\section{VELOCITY ANISOTROPY}

We have taken the results from the 3D-MHD simulations and produced 2D maps of the mean velocity and centroids of velocity, integrating along each of the cardinal $(x, y, z)$ directions. We consider an isothermal, 
optically thin medium, with an emissivity linearly proportional to the density (e.g. cold H I). If we integrate along the $x$-axis we can obtain a $2 \mathrm{D}$ map of the mean velocity perpendicular to the $y-z$ plane:

$$
V_{x}(y, z)=\frac{1}{N_{x}} \int v_{x}(x, y, z) d x,
$$

where $N_{x}$ is the number of cells used to discretize $x$, and $v_{x}$ is the projection of the velocity field along $\hat{\mathbf{x}}$. This mean LOS velocity traces the velocity structure in a similar manner as the column density follows the density structure (Esquivel \& Lazarian 2005, henceforth EL05). However, it cannot be obtained directly from observations.

In real observations we are faced with a density weighted mean (i.e. the velocity centroids). For the centroid maps we used their conventional (normalized) form, under the assumption of an optically thin medium whose emissivity is proportional to the density (see EL05: Esquivel et al. 2007):

$$
C_{x}(y, z)=\frac{\int v_{x}(x, y, z) \rho(x, y, z) d x}{\int \rho(x, y, z) d x} .
$$

Analogous expressions to equations (4) and (5) can be used to obtain the mean velocity, or velocity centroids with the LOS aligned with $\hat{\mathbf{y}}$, or $\hat{\mathbf{z}}$.

The two-point, second-order structure function of a quantity $f(\mathbf{x})$ is defined as:

$$
S F(\mathbf{r})=\left\langle[f(\mathbf{x})-f(\mathbf{x}+\mathbf{r})]^{2}\right\rangle,
$$

where $\langle\ldots\rangle$ denotes an ensemble average over all space ( $\mathbf{x}$ ), and $\mathbf{r}$ is the separation or "lag". A closely related measure is the correlation function $C F(\mathbf{r})=\langle f(\mathbf{x}) f(\mathbf{x}+\mathbf{r})\rangle$, which differs from the $S F$ basically by a constant (see for instance EL05). For the sake of simplicity we will use the following notation: $S F_{\mathrm{V}, \mathrm{x}}(\mathbf{R})$, and $S F_{\mathrm{C}, \mathrm{x}}(\mathbf{R})$ denote the structure function applied to a map of mean velocity (eq. 4), and a map of velocity centroids (eq. 5), respectively. The lag $\mathbf{R}$ is written in upper case letters to denote that is a twodimensional vector, while the $x$ in the sub-index indicates that the mean velocity or centroids were obtained integrating along the $x$-axis [in which case $\mathbf{R}=(y, z)$ ]. The power-spectrum is another recurrent tool in turbulence studies. It is the Fourier transform of the correlation function, thus it provides equivalent information.

If the turbulence were isotropic these two point statistics would (statistically) not depend on the direction of the lag, or on the direction of the wave number for the power-spectrum. In fact it is customary to assume isotropy and average over all angles to present just a radial dependence of $S F(r)$. It is well known, however, that the magnetic fields breaks the isotropy and the turbulence becomes anisotropic (see Higdon 1984 and references therein, GS95, Cho \& Lazarian 2005 for a review).

\subsection{Additional Density Fluctuations}

Spectroscopic observations are sensitive to density and velocity fluctuations simultaneously. For the particular case of centroid maps, it was clear for instance that the strong fluctuations in highly supersonic turbulence $\left(M_{s} \gtrsim 2.5\right)$ affected severely our ability to determine the spectral index of velocity (Lazarian \& Esquivel 2003 EL05, Esquivel et al. 2007).

In order to further study the impact of density fluctuations in the anisotropy that can be observed we study four different maps obtained for each of the simulations of Table 1. Firstly, a map of the mean velocity obtained as in equation 4, which have information exclusively of velocity, but can not be obtained from observations. Secondly, a map of velocity centroids (eq. 5), obtained with the density field as obtained from the simulations. Thirdly, another map of velocity centroids, but in this case modulating the density by an $r^{-2}$ profile. The direction of the gradient is $(\hat{x}, \hat{y}, \hat{z})$, so that it is oblique to both the LOS and the mean B field. To avoid the singularity at $r=0$ we have placed the origin half a pixel outside the computational domain, and the resulting density field was rescaled to have a mean value of 1 . This gradient introduce a large-scale density variation, which could be encountered in observations of the ISM, but which is a not a product of turbulence (e.g. self-gravity). And fourthly, another map of centroids was obtained with a density field to which we have added white noise. We model the noise using fractional Brownian motion (fBm) fractal structures, that can be characterized by a power spectrum index (Stutzki et al. 1998; Bensch et al. 2001), which for white noise we have set to zero (Hat power spectrum). The resulting data cubes have a Gaussian probability distribution function. Their dispersion have been scaled to the same value of the original density for each of the models. The noise is added to the density, and to preserve the density positive defined we have set a floor value of 0.01 (the mean density is 1.0 ).

The resulting density fields are illustrated in Fig 1. where we present map of the $x y$-midplane cut of the density of one of the models (M8, super-sonic and subAlfvénic, see Table 11). The first panel (a) displays the original density, the middle panel (b) shows the effect of the large scale gradient, and in (c) the addition of white noise is evident.

In the following section we will study the correlation and structure functions of the maps of mean velocity and of centroids, and how their anisotropy depends on the parameters of the models.

\section{RESULTS}

Observations sample the entire LOS, and at a given velocity one has contribution of material that could be anywhere along that LOS. In some sense, one can say that observations average the information in the position perpendicular to the plane of the sky. Thus, from an observational point of view, it is more natural to study the anisotropy in a global frame, namely, the anisotropy with respect to the direction of the mean magnetic field (as opposed to the local magnetic field, from which a GS95 scaling is retrieved).

We have shown (LPE02, Esquivel et al. 2003, EL05) that in such a global frame indeed the statistics of velocity centroids reveal the direction of the mean magnetic field. In Figure 2 we present contours of equal correlation in one of the models (M8, the same used in Figure 1). The mean LOS contours are remarkably similar to those of velocity centroids.In the figure we only show the results for the centroids with the original density because they are very similar to those obtained if the $\propto r^{-2}$ den- 

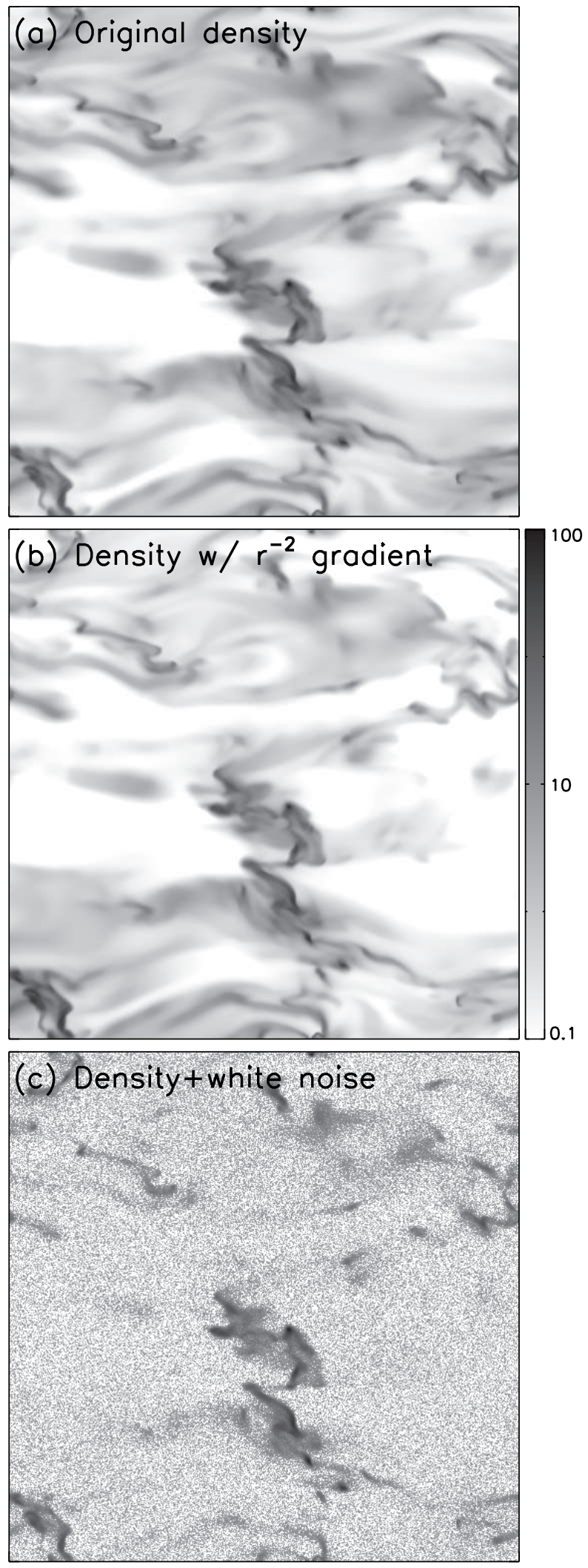

FIG. 1.- Density cuts in the $X Y$-midplane. (a) Original density, (b) density modulated by an $\propto r^{-2}$ gradient (decreasing from the left to the right, and from the bottom to the top), and (c) the density with additional white noise. All the plots are in the same (logarithmic) scale, as indicated by the bar on the right. sity gradient, or white noise (see 3.1 ) are included.

In panels (a) and (c) in Figure 2 the line of sight is in the direction parallel to the mean magnetic field $(x)$, thus the two axes shown are perpendicular to it, and the contours are more or less circular (isotropic). Panels (b) and $(\mathrm{d})$ have been integrated in a direction perpendicular to the mean plane $(z)$ and the resulting correlations show a clear anisotropy in the direction of the B field, the same result is obtained if we integrate along the $y$-axis. This result about the direction of the mean magnetic field (or more accurately, its projection onto the plane of the sky) is quite robust.

To address the dependence of the anisotropy with scale, we start by defining a simple measure of the degree of anisotropy: the ratio of the structure function in the two directions that are perpendicular to the LOS (e.g. $\left.S F_{C, z}(x, 0) / S F_{C, z}(0, y)\right)$, which we have computed for all the models. In Figure 3 we present an example obtained from the same model of the previous two Figures. In the top three panels $(\mathrm{a}-\mathrm{c})$ we show the results of the mean LOS velocity, and in the bottom panels (d-f) the results with the different velocity centroids.

If this ratio is one the structure function is isotropic, as in the case of panels (a) and (d), which correspond to the structure functions when the mean magnetic field and the LOS are aligned. The rest of the panels are clearly anisotropic, with a degree of anisotropy, whose exact value depended on model. In Figure 3 we indicated with vertical lines the scale length of injection (turbulence forcing) with a dotted line. At such large scales, and down to about $1 / 5$ of the box size (marked with a vertical dashed line) the effect of the forcing is evident. For the mean LOS velocity, and the centroids with the original density field, we found that the anisotropy was virtually scale independent from the small scales up to separations on the order $\sim 1 / 5$ of the computational box (half the size of the injection scale). For centroids with additional density fluctuations (dashed and dotted lines) we see that the anisotropy shows a flat (scale independent) behavior for separations $\gtrsim 10$ and up to $\sim 1 / 5$ of the computational box. In other words, the additional density fluctuations interfere with the measured anisotropy degree at the smallest scales, making the statistics more isotropic.

More interesting than confirming the scale independence on the structure functions in the inertial range of the turbulent cascade (there is already evidence of this in CLV02), it is to study how the degree of anisotropy depends on the turbulence parameters (sonic and Alfvénic Mach numbers at the injection scale). To do this we have calculated the degree of anisotropy on all the models, as exemplified for model M8 in Fig. 3 and computed the average value in scales between 10 grid points and $L / 5$. Below 10 grid points scale, the density from the MHD simulations is severely affected by numerical diffusion, and the effect of the large scale density gradient and noise are more pronounced. The results are condensed in Figure 4, where we have plotted the average degree of isotropy as a function of the sonic Mach number and of the Alfvénic Mach number. The Mach numbers are indicated by the different symbols (and colors in the online version) as displayed in the legend. The error bars show the variations while obtaining the average value. They are the product of differences in the two possible lines of 

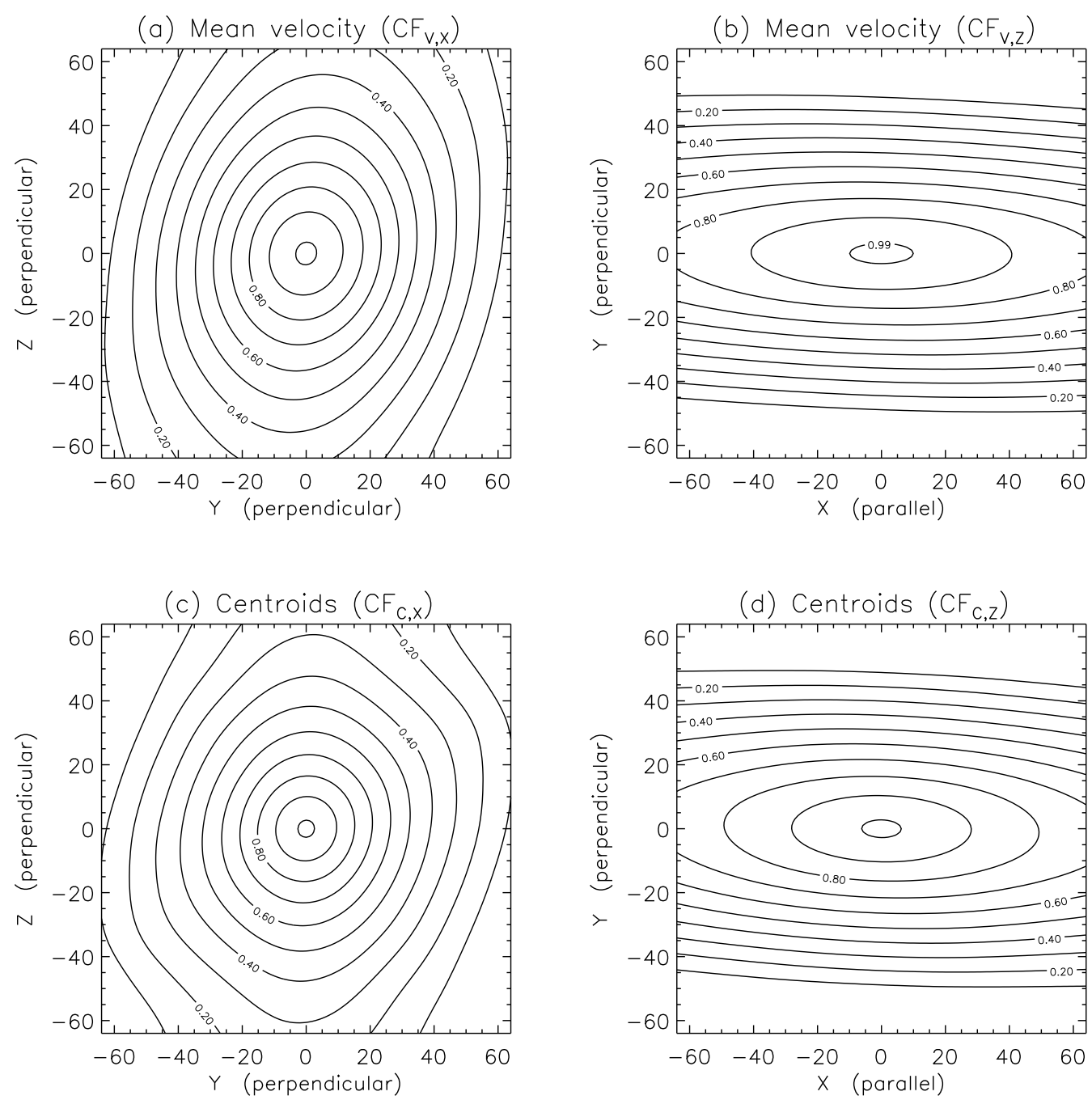

FIG. 2.- Example of the iso-contours of the correlation function in one of the simulations (model M8: super-sonic and sub-Alfvénic). The first two panels [(a) and (b)] correspond to correlations in mean velocity maps, the last two [(c) and (d)] are correlations in velocity centroid maps (with the original density, see $\$ 3.1$ ). In the plots on the left column [panels (a) and (c)] the LOS is parallel to the mean B field. In the right column [panels (b) and (d)] the LOS is perpendicular to the mean B field, which is in this case aligned with the horizontal axis.

sight ( $y$ - and $z$-axis) and slight scale variations.

One can see from Figure 4 that the centroids maps without noise or gradients have the smallest error bars, thus the least dependence on scale. The rest of the centroids and the mean LOS velocity have slightly larger error bars, and therefore some (small) scale dependence. It is also quite noticeable that, the results for the different centroids (with the original density or modified data) are very similar. This is not too surprising, because we have restricted ourselves to scales that were seen as less effected by either the $\propto r^{-2}$ gradient or the noise. However, to apply this technique to real data it would be advisable to calculate the anisotropy degree on several scales and search for a scale independent range, in the same manner one restricts the inertial range looking for power-laws when analyzing power spectra.

It is clear also, that the degree of anisotropy depends mostly on the Alfvénic Mach number: for increasing values of the magnetic field the level of anisotropy increases as well (smaller $S F_{\perp} / S F_{\|}$). For the velocity centroids we have the same general trend, but not as pronounced. For instance, for $M_{A} \gtrsim 1.4$ the degree of anisotropy is barely distinguishable from isotropic, while in the mean LOS velocity map this was only the case for a very weak B field $\left(M_{A} \sim 7.5\right)$. Centroids also show a weak dependence on $M_{s}$, but only for moderate to low magnetizations $\left(M_{A}>0.4\right)$. For strong magnetic fields $\left(M_{A} \sim 0.4\right)$ we observe some dependence on the sonic Mach number. Since this was not noticeable in Fig.4 (a), and given the similarity of Figs. 4(b-d), one can attribute such dependence to the original density field (i.e. arising from shocks in supersonic turbulence). This strong influence of the sonic Mach number is seen as a positive slope for the most magnetized simulations.

The results of a clean dependence on the Alfvénic Mach number and a weak dependence on $M_{s}$ (for small to moderate magnetic field strengths), are encouraging. While the sonic Mach number can be obtained by a variety 


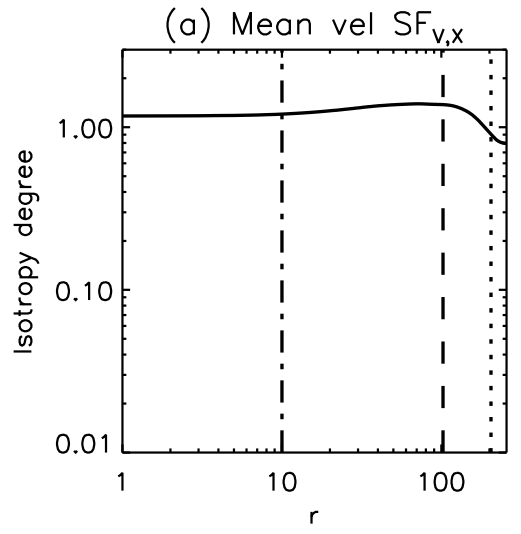

(d) Vel centroids SF $F_{c, x}$

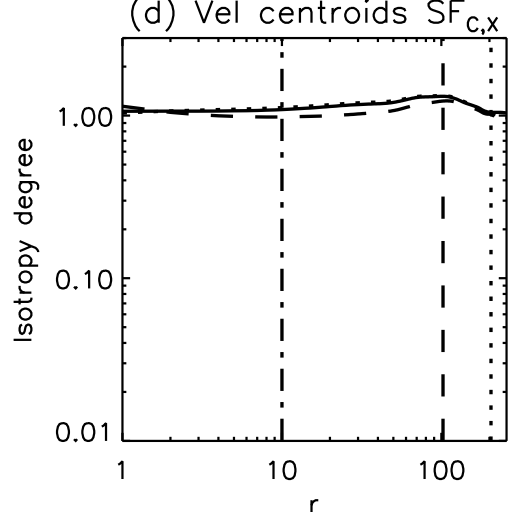

(b) Mean vel $\mathrm{SF}_{\mathrm{V}, \mathrm{Y}}$

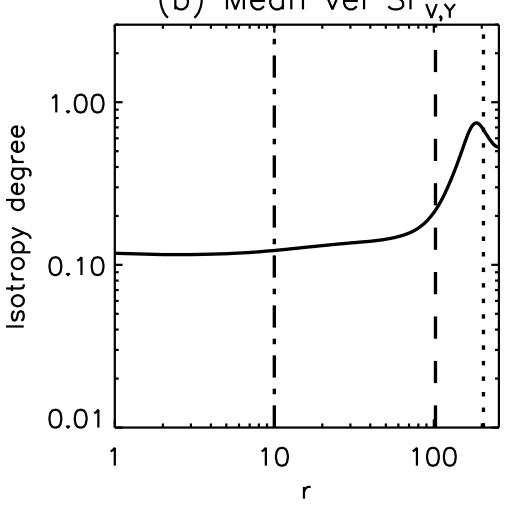

(e) Vel centroids $S_{C, Y}$

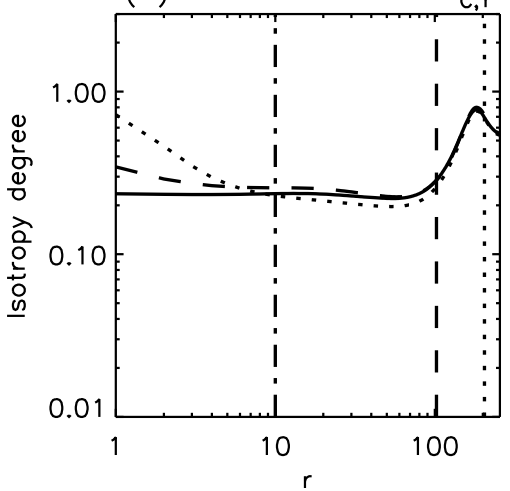

(c) Mean vel $\mathrm{SF}_{\mathrm{v}, \mathrm{z}}$

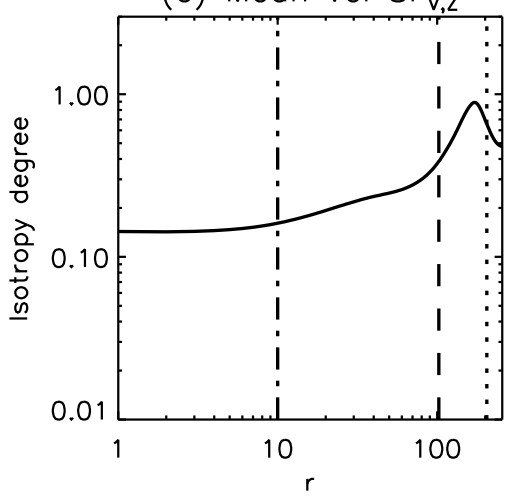

(f) Vel centroids $S F_{C, Z}$

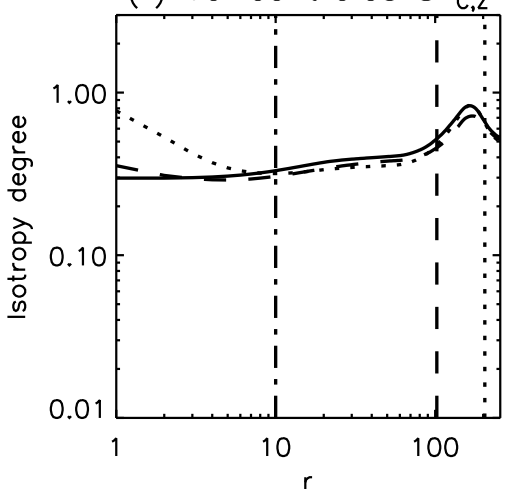

Fig. 3.- Example of the degree of anisotropy of the structure functions in the same model shown in Figure 2 as observed from different directions (a value of 1 means isotropic). The top row [panels (a)-(c)] are obtained with the mean density maps while the bottom row $[(\mathrm{d})-(\mathrm{f})]$ with maps of velocity centroids. The different lines in panels (d-f) denote the density field used to obtain the centroids, the solid line corresponds to the original density, the dashed line to the $\propto r^{-2}$ gradient, and the dotted line to the addition of white noise. The LOS is aligned with the $x$-axis (parallel to the B field) in the left column [(a) and (d)], with the $y-$ axis in the middle column [(b) and (e)], and with the $z$-axis in the right column [(c) and (f)].

of techniques (Padoan et al. 1997, Passot \& VázquezSemadeni 2003; Kowal et al. 2007; Federrath et al. 2008; Burkhart et al. 2009), the Alfvénic Mach number has remained elusive. We are confident that anisotropy studies, along with the Chandrasekhar-Fermi technique (see for instance Falceta-Gonçalves et al. 2008) are starting to change this situation.

All in all, the observable turbulent fluctuations, as represented by velocity centroids, are sensitive to the fluid magnetization given by the Alfvénic Mach number $M_{A}$. The dependence of the anisotropy on the sonic Mach number $M_{s}$ is, however, not always negligible. The latter number can be obtained using other statistics studied in the literature (see Kowal et al. 2007, Burkhart et al. 2009, 2010, Esquivel \& Lazarian 2010)

\section{DISCUSSION AND SUMMARY}

Advances in understanding of the nature of magnetized turbulence drive the development of the techniques to study turbulence through observations. For these studies different approaches can (and should) be used. However, it is important to understand what is the maximal information that one can get from observations.

Recently observational studies of interstellar anisotropies have been performed by Heyer et al. (2008). The resulting anisotropies were broadly consistent with the expectations obtained in theoretical and numerical studies (see LPE02, EL05), revealing the direction of the mean magnetic field. Both theoretical predictions and numerical calculations, including those in the present paper indicate that the anisotropy expected in the global frame of reference, which is sampled in the observations, should have a range in which they do not depend on the scale. In addition, we pay attention to the dependence of the velocity anisotropy on the sonic Mach numbers.

In general, the measures we use to study the anisotropy may be affected by other interfering factors. For instance, density effects can affect velocity centroid measures. Attempts to mitigate the effect fluctuating density field on velocity centroids was attempted in Lazarian \& Esquivel (2003) where new measures termed "modified centroids" were introduced. However, further studies in Esquivel \& Lazarian (2005); Esquivel et al. (2007) revealed that the "modified centroids" improve the representation of turbulent velocity only at moderate $M_{s}$. We have also used MVCs for the models presented in this paper and found that the results were actually worse (noisier) than with ordinary centroids. Thus in the present study of anisotropy we present only ordinary centroids.

To study the robustness of the method we have added fluctuations to the density field and reanalyze the anisotropy of centroids obtained with these new data. We find that the range of scales in which the anisotropy 

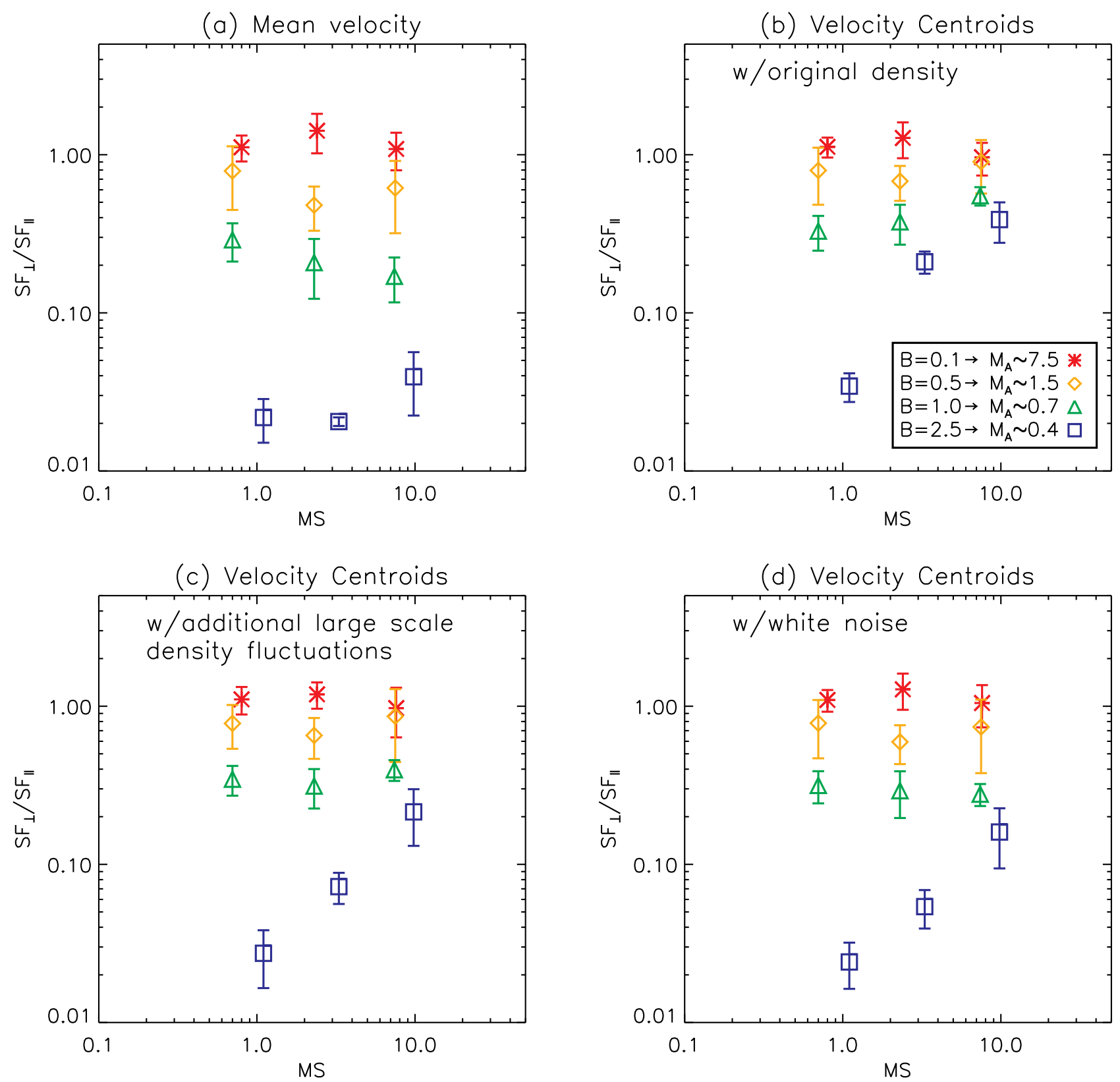

Fig. 4.- Degree of anisotropy in all the models averaged over scales from 10 grid points to $1 / 5$ of the computational box. The horizontal axis corresponds to the sonic Mach Number, the Alfvénic Mach Number is indicated by the various symbols (and colors in the online version) as shown in the label. In the left panel (a) we plotted the anisotropy in velocity centroids, in the right panel (b) the anisotropy in the average LOS velocity. In both figures the results are obtaining averaging the two cases where the LOS is perpendicular to the mean field. The error bars show the maximum variation of the averaging procedure (including variation across scales).

degree is scale independent is limited to separations smaller than the injection scale (in fact smaller than half of the injection scale), but at the same time large enough to avoid the effects of the additional fluctuations. In our models we have found that the effect of an $\propto r^{-1}$ gradient and white noise can be avoided by setting the smallest scale used to measure the anisotropy to 10 cells. With real observations one has to measure the anisotropy and determine the appropriate range.

Heyer et al. (2008), use not centroids, but a Principal Component Analysis (PCA) to study turbulence through observations. PCA analyzes the data in position- position-velocity (PPV) space which could potentially have some useful information that is lost in the averaging procedure in velocity centroids. However, PCA relies in calibration from numerical models, while structure functions and centroids can be described analytically and predictions of their results follow directly from theory.

Our simulations show a dependence on the sonic Mach number which was not reported by Heyer et al. (2008). We suspect the reason is that dependence on $M_{s}$ is less prominent compared to the one on $M_{A}$. However, if one wants a more precise analysis it should be taken into account. In spite of this, we should stress the pio- 
neering significance of the observational studies of Heyer et al. (2008) which moved this technique of magnetic field study from theoretical and numerical domain (see LPE02, EL05) to a domain of practical application. We believe that the technique has great future and view this paper as a contribution to its improvement.

We have taken a set of simulations of fully-developed, driven MHD turbulence with different combinations of sonic and Alfvénic Mach numbers to study the velocity anisotropy available from observations.

Our results can be summarized as follows:

- Synthetic maps obtained through compressible MHD simulations reveal a clear anisotropy of the velocity field, in alignment with the direction of the mean magnetic field. This is true for Alfvénic Mach numbers less than $M_{A} \simeq 1.5$.

- The anisotropy, measured at scales in the inertial range of the turbulent cascade is scale independent in the global frame of reference, as opposed to the scale dependent anisotropy with is obtained with respect to the the direction of the local magnetic field.
- The degree of anisotropy is dominated by the Alfvénic Mach number. However, a dependence on the sonic Mach number gets prominent for highly magnetized gas. The anisotropy in velocity centroids differs from that of the mean velocity. As many other statistics are sensitive to the sonic Mach number this allows to make a correction for $M_{s}$ to retrieve $M_{A}$.

- To show that the method is robust against largescale density fluctuations and noise, we have obtained centroids with modified density fields, to which we have added an $\propto r^{-2}$ gradient, or white noise. We see that these have an effect that is mostly seen at the smallest scales. If the degree of anisotropy is measured avoiding such scales the results remained basically unchanged.

AE acknowledges support from grants CONACyT 61547, 101356, and 101975. AL acknowledges the NSF grant AST 0808118 and the support of the Center of Magnetic Self-Organization (CMSO).

\section{REFERENCES}

Ballesteros-Paredes, J., Klessen, R. S., Mac Low, M.-M., \& Vazquez-Semadeni, E. 2007, in Protostars and Planets V, ed. B. Reipurth, D. Jewitt, \& K. Keil, 63-80

Bensch, F., Stutzki, J., \& Ossenkopf, V. 2001, A\&A, 366, 636

Beresnyak, A., \& Lazarian, A. 2010, ApJ, 722, L110

Burkhart, B., Falceta-Gonçalves, D., Kowal, G., \& Lazarian, A. 2009, ApJ, 693, 250

Burkhart, B., Stanimirović, S., Lazarian, A., \& Kowal, G. 2010, ApJ, 708, 1204

Cho, J., \& Lazarian, A. 2002, Physical Review Letters, 88, 245001 -. 2003, MNRAS, 345, 325

- 2005, Theoretical and Computational Fluid Dynamics, 19, 127

Cho, J., Lazarian, A., \& Vishniac, E. T. 2002, ApJ, 564, 291

Cho, J., \& Vishniac, E. T. 2000, ApJ, 539, 273

Elmegreen, B. G., \& Scalo, J. 2004, ARA\&A, 42, 211

Esquivel, A., \& Lazarian, A. 2005, ApJ, 631, 320

-. 2010, ApJ, 710, 125

Esquivel, A., Lazarian, A., Horibe, S., Cho, J., Ossenkopf, V., \& Stutzki, J. 2007, MNRAS, 381, 1733

Esquivel, A., Lazarian, A., Pogosyan, D., \& Cho, J. 2003 MNRAS, 342, 325

Falceta-Gonçalves, D., Lazarian, A., \& Kowal, G. 2008, ApJ, 679 537

Federrath, C., Klessen, R. S., \& Schmidt, W. 2008, ApJ, 688, L79

Goldreich, P., \& Sridhar, S. 1995, ApJ, 438, 763

Heyer, M., Gong, H., Ostriker, E., \& Brunt, C. 2008, ApJ, 680, 420

Higdon, J. C. 1984, ApJ, 285, 109
Jiang, G.-S., \& Wu, C.-C. 1999, Journal of Computational Physics, 150, 561

Kowal, G., \& Lazarian, A. 2010, ApJ, 720, 742

Kowal, G., Lazarian, A., \& Beresnyak, A. 2007, ApJ, 658, 423

Lazarian, A., \& Cho, J. 2005, Physica Scripta Volume T, 116, 32

Lazarian, A., \& Esquivel, A. 2003, ApJ, 592, L37

Lazarian, A., Pogosyan, D., \& Esquivel, A. 2002, in Astronomical Society of the Pacific Conference Series, Vol. 276, Seeing Through the Dust: The Detection of HI and the Exploration of the ISM in Galaxies, ed. A. R. Taylor, T. L. Landecker, \& A. G. Willis, 182-+

Lazarian, A., \& Vishniac, E. T. 1999, ApJ, 517, 700

Lithwick, Y., \& Goldreich, P. 2001, ApJ, 562, 279

Mac Low, M.-M., \& Klessen, R. S. 2004, Reviews of Modern Physics, 76, 125

Maron, J., \& Goldreich, P. 2001, ApJ, 554, 1175

McKee, C. F., \& Ostriker, E. C. 2007, ARA\&A, 45, 565

Montgomery, D. 1982, Phys. Scr, 2, 83

Padoan, P., Jones, B. J. T., \& Nordlund, A. P. 1997, ApJ, 474, 730

Passot, T., \& Vázquez-Semadeni, E. 2003, A\&A, 398, 845

Shu, C., \& Osher, S. 1989, Journal of Computational Physics, 83, 32

Stutzki, J., Bensch, F., Heithausen, A., Ossenkopf, V., \&

Zielinsky, M. 1998, A\&A, 336, 697

Tóth, G. 2000, Journal of Computational Physics, 161, 605

Vestuto, J. G., Ostriker, E. C., \& Stone, J. M. 2003, ApJ, 590, 858 